\title{
3D Numerical Simulations of Negative Hydrogen Ion Extraction Using Realistic Plasma Parameters, Geometry of the Extraction Aperture and Full 3D Magnetic Field Mapa)
}

\author{
S. Mochalskyy, ${ }^{1, b)}$ D. Wünderlich, ${ }^{1}$ B. Ruf, ${ }^{1}$ P. Franzen, ${ }^{1}$ U. Fantz ${ }^{1}$ and T. Minea ${ }^{2}$ \\ ${ }^{1}$ Max-Planck-Institut für Plasmaphysik, EURATOM Association, Boltzmannstr. 2, D-85748, Garching, Germany \\ ${ }^{2}$ Laboratorire de physique des gaz et des plasma,CNRS, University Paris-Sud 11, 15 rue G. Clemenceau, F91405, France
}

(Presented XXXXX; received XXXXX; accepted XXXXX; published online XXXXX)

(Dates appearing here are provided by the Editorial Office)

\begin{abstract}
Decreasing the co-extracted electron current while simultaneously keeping negative ion (NI) current sufficiently high is a crucial issue on the development plasma source system for ITER Neutral Beam Injector. To support finding the best extraction conditions the 3D PIC MCC electrostatic code ONIX has been developed. Close collaboration with experiments and other numerical models allows performing realistic simulations with relevant input parameters: plasma properties, geometry of the extraction aperture, full $3 \mathrm{~d}$ magnetic field map etc. For the first time ONIX has been benchmarked with commercial positive ions tracing code KOBRA3D. A very good agreement in terms of the meniscus position and depth has been found. Simulation of NI extraction with different e/NI ratio in bulk plasma shows high relevance of the direct negative ion extraction from the surface produced NI in order to obtain extracted NI current as in the experimental results from BATMAN testbed.
\end{abstract}

\section{INTRODUCTION}

Neutral Beam Injection (NBI) will play an essential role for heating and current drive of the future experimental thermonuclear reactor ITER. In order to satisfy ITER requirements neutral beam must be injected with energies of order $1 \mathrm{MeV}$ [1]. Such high energetic neutral beam can be obtained only by means of the production, extraction and further acceleration of negative ions. Thus, to reach ITER condition the NI source should supply 69A extracted current in hydrogen and 57A in deuterium plasma.

Negative ions (NI) are generated in the low pressure $(\mathrm{p}<0.6 \mathrm{~Pa})$ Radio-Frequency (RF) plasma source - Fig.1. The system consists of three main parts: (i) "driver", where the plasma $\left(\mathrm{Te} \approx 10 \mathrm{eV}, \mathrm{n}_{\mathrm{e}} \approx 10^{18} \mathrm{~m}^{-3}\right.$ ) is produced via $\mathrm{RF}$ power (up to $100 \mathrm{~kW}, \sim 1 \mathrm{MHz}$ ) coupled to the gas; (ii) "expansion region" - here plasma diffuses and electrons are cooled $(\mathrm{Te} \sim 1 \mathrm{eV})$ by a magnetic filter; (iii) "extraction region", where produced negative ions are extracted through two multi-aperture grids ( $\mathrm{PG}$ - plasma grid and EG - extraction grid). A positive extraction potential $(\sim 10 \mathrm{kV})$ is applied between them to drain out NI and repel positively charged particles [2]. In order to increase the amount of produced and extracted NI, Cesium vapour is injected in the source. Cesium covers the surface of the plasma grid and NI are produced via surface conversion of

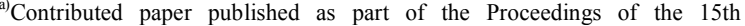
International Conference on Ion Source, Chiba, Japan, September, 2013 ${ }^{b)}$ Author to whom correspondence should be addressed. Electronic mail: serhiy.mochalskyy@ipp.mpg.de.
}

atoms and positive ions [3] where the atoms are dominant specie. One of the main issues of the source is suppression of the co-extracted electrons. They are dumped on the extraction grid surface by means of the deflecting field, which is produced via the magnets embedded inside this grid. In order to avoid melting of the extraction grid, the ratio between extracted electron and NI current has to be kept below 1 .

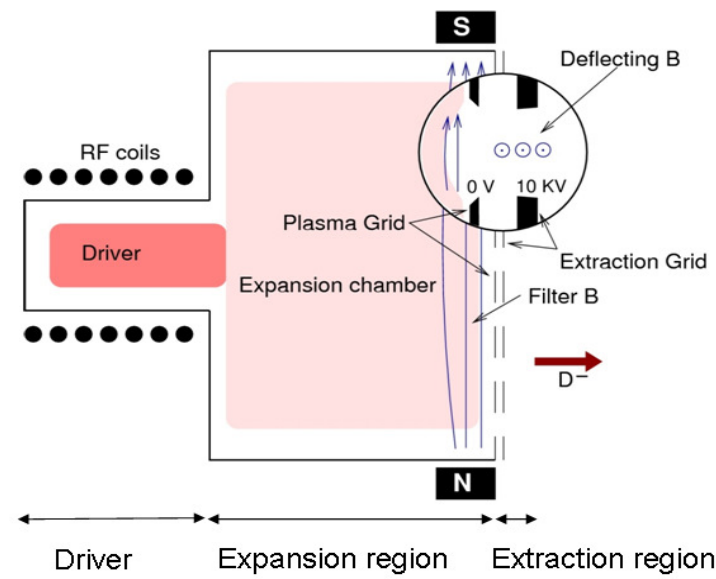

FIG. 1. Schematic view of the ITER NBI negative ion plasma source prototype designed at IPP.

Nowadays, there are two ITER-like NI source test bed machines in operations in IPP Garching: BATMAN [4] is a $1 / 8$ ITER size NI source test bed and ELISE is a $1 / 2$ ITER 
size source that is the largest radio frequency NI source in operation at present [5].

This contribution focuses on the modeling and crosschecking analysis of the extracted NI and electron current from the BATMAN testbed. The proximity with experiments allows performing realistic simulation with qualitative description of the computation domain.

\section{SIMULATION MODEL}

The 3D Particle-in-Cell Monte Carlo Collision electrostatic code ONIX (Orsay Negative Ion eXtraction) has been initially developed in laboratory LPGP, Orsay, France to simulate negative ion production and their extraction in ITER-like NI source extraction region [6]. The code uses Cartesian coordinates and can deal with complex boundary geometry as in the case of the extraction aperture. In order to speed up the calculation the code is parallelized via Message Passing Interface using full spatial domain and particle decomposition techniques. Detailed description of the code together with collision module can be found in [7]. Here will be shown only the recent development of the ONIX code and underlying the differences with previous version.

The simulation domain is shown in Fig. 2. It includes one typical orifice of the plasma grid with dimensions as in BATMAN testbed - $8 \mathrm{~mm}$ diameter, $4 \mathrm{~mm}$ width and double chamfered edges with the angle of 45 degree from each side. The periodic boundary condition is used in horizontal " $y$ " and vertical " $z$ " directions representing an infinity array of PG apertures. The extraction potential of $9 \mathrm{kV}$ is applied to the right boundary of the domain, when the left hand side boundary as well as the walls of PG grid are $0 \mathrm{~V}$. The volume plasma parameters used in the simulation were taken from different diagnostic installed at BATMAN. Density ne: $\mathrm{n}_{\mathrm{H}}^{-}$(volume): $\mathrm{n}_{\mathrm{H}}^{+}: \mathrm{n}_{\mathrm{H} 2}{ }^{+}: \mathrm{n}_{\mathrm{H} 3}{ }^{+}=2.7$ : 0.3: 1.2: 1.2: $0.3 \times 10^{17} \mathrm{~m}^{-3}$; temperature Te: $\mathrm{T}_{\mathrm{H}}^{-}$(volume): $\mathrm{T}_{\mathrm{H}}{ }^{+}: \mathrm{T}_{\mathrm{H} 2}{ }^{+}: \mathrm{T}_{\mathrm{H} 3}{ }^{+}=2.0: 0.1: 0.8: 0.1: 0.1 \mathrm{eV}$.

In order to decrease numerical noise and perform more realistic study the second order charge projection routine [8] onto the PIC grid nodes has been implemented. Such algorithm better describes the high density plasma in PIC simulation significantly reducing the errors.

NI production at the Cs covered PG surface was implemented in the code as an arbitrary negative ion flux $(660 \mathrm{~A} / \mathrm{m} 2)$, which has been taken from the simulation of 1D Bacon code [9]. Negative ions are uniformly launched over the PG surface with random initial angle and energy between $0.1-1 \mathrm{eV}$.

From the previous study [7] it was found that small changes in the external applied filter and deflecting magnetic fields cause an important effect on the coextracted electron current. Therefore, the former uniform distribution of the magnetic field along the horizontal and vertical direction has been replaced by the realistic full 3D magnetic field map calculated for the central aperture of the PG.

\section{RESULTS AND DISCUSSIONS}

\section{A. Extracted NI and electron current}

The ONIX code is able not only to calculate the extracted NI current, but can also determine the origin of the produced NI. The dominant process is the NI production at $\mathrm{Cs}$ covered $\mathrm{PG}$ chamfered surface. The extracted NI current density from this channel is $\sim 14.9$ $\mathrm{mA} / \mathrm{cm}^{2}$. A negative reverse sheath [7] $(\sim-6 \mathrm{~V})$ in the front of PG flat surface reflects most of NI back towards the PG wall, where they are destroyed. Therefore, the extracted current density from this channel is very low $(\sim 0.4$ $\left.\mathrm{mA} / \mathrm{cm}^{2}\right)$. The extracted current from the volume produced NI is about 10 percent of the surface production - 1.6 $\mathrm{mA} / \mathrm{cm}^{2}$. The total extracted NI current density is $\sim 16.9$ $\mathrm{mA} / \mathrm{cm}^{2}$ that is in good agreement with experimental results obtained at BATMAN test bed $\left(\sim 14 \mathrm{~mA} / \mathrm{cm}^{2}\right)$ for a typical set of plasma parameters. The co-extracted electron current density $\left(\sim 79 \mathrm{~mA} / \mathrm{cm}^{2}\right)$ is in $\sim 4.5 \pm 0.5$ times higher than NI. In experimental measurements the similar e/NI current ratio has also been found $\sim 3$ during performance with very low $(1.4 \mathrm{~V})$ electron suppression bias potential applied to the plasma grid.

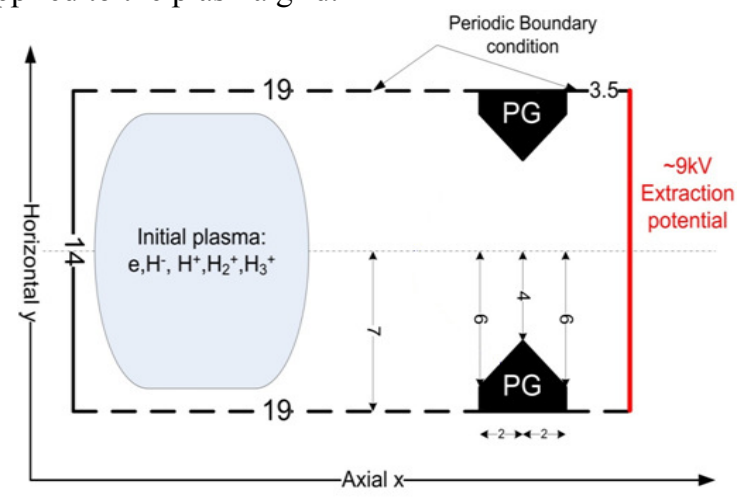

FIG. 2. Schematic view of the simulation domain used in ONIX.

\section{B. Meniscus formation}

The "meniscus" position and the deepness of its curvature play an important role for the NI beam formation and beam optics. In order to validate the correctness of the ONIX "meniscus" calculation the code has been benchmarked with the commercial 3D transport code for positive ion extraction KOBRA3D [10]. The ONIX code has been modified and adapted to simulate PI extraction. The codes calculate the "meniscus" position in two different ways. ONIX uses in a self-consistent manner the initial plasma density $\left(0.8 \cdot 10^{17} \mathrm{~m}^{-3}\right)$ to calculate the extracted $\mathrm{H}^{+}$current, potential and steady state density distribution of each species; KOBRA3D (PI test particle tracking code) calculates the potential profile using the PI extracted current as the input parameter. Fig. 3 shows the potential distribution in vicinity to the $\mathrm{PG}$ aperture from both models. The perfect agreement is found for $-50 \mathrm{~V}$ (red) and $-10 \mathrm{~V}$ (black) potential isolines. The line $1 \mathrm{~V}$ 
(green) is slightly shifted ( $\sim 0.2 \mathrm{~mm})$ marking the range of the error bar.

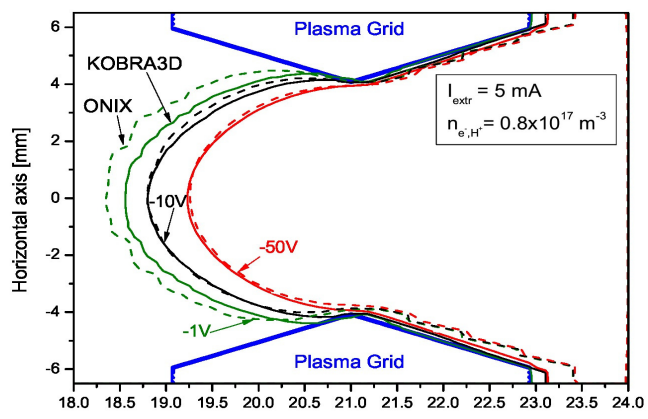

Beamaxis $[\mathrm{mm}]$

FIG. 3.Potential distribution from ONIX (dashed lines) and KOBRA3D (solid line) simulations.

After the ONIX code has been partially validated and benchmarked against the KOBRA3D code, realistic simulations on the plasma "meniscus" in NI extraction ONIX version were performed. Fig. 4 represents the $\mathrm{H}+$ density distribution close to PG aperture in the simulation using the plasma parameters given in Section II for NI extraction. The plasma "meniscus" penetrates into the expansion chamber with the deepest edge $\sim 2.5 \mathrm{~mm}$ $(x=16.5 \mathrm{~mm})$ from the beginning of $P G$ wall $(x=19.1 \mathrm{~mm})$.

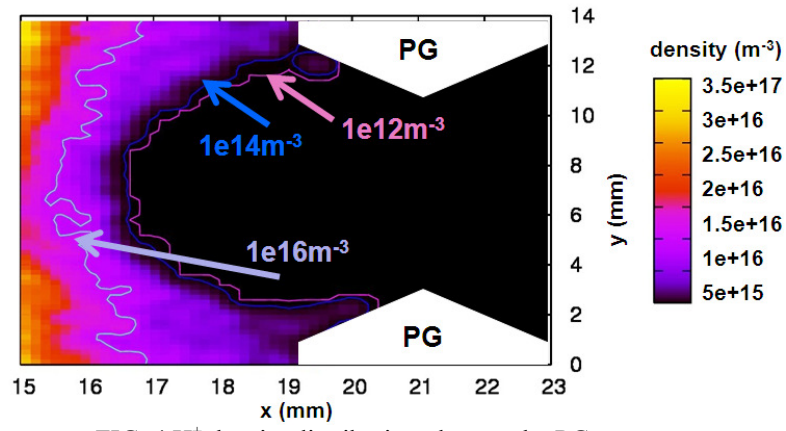

FIG. $4 . \mathrm{H}^{+}$density distribution close to the PG aperture.

\section{Ion-ion plasma simulation}

Measurements of $\mathrm{H}^{-}$density on BATMAN testbed by means of Cavity Ring Down Spectroscopy have shown significant increasing amount of NI close to the extraction system $(\sim 2 \mathrm{~cm}$ from the $\mathrm{PG})$ in experiments with surface negative ion production. The NI density grows from $\mathrm{nH}-$ $\sim 10^{16} \mathrm{~m}^{-3}$ in Cs free regime to $\mathrm{n}_{\mathrm{H}}^{-} \sim 10^{17} \mathrm{~m}^{-3}$ for surface production experiments [11]. Such experimental results are in disagreement with ONIX simulations, where most of the produced NI from the PG surface are either directly extracted towards the extraction grid or immediately repelled back towards the PG wall if the local field is reversed [7]. Only a small fraction of NI could overcome this field and penetrate the plasma volume. The simulation shows total NI density in the bulk plasma $\sim 5 \cdot 10^{16} \mathrm{~m}^{-3}$. To better understand the influence of a growing NI density into the plasma volume on the total extracted NI current, the parametric study of different electronegative bulk plasmas (ratio between electron and NI density) has been performed. Fig. 5 shows the extracted NI current density from the simulation of plasma containing e/NI ratio from $95 \%$ electrons and 5\% NI (blue line) to $95 \%$ NI and $5 \%$ electrons (red line). The simulations show that even with complete ion-ion plasma the extracted NI current density from the bulk plasma stays rather low $\left(\sim 9.5 \mathrm{~mA} / \mathrm{cm}^{2}\right)$. Moreover, it was found that e/NI current ratio reaches $\sim 1$ when the initial plasma will consist of minimum $\sim 90 \%$ of NI and only $\sim 10 \%$ of electrons that is far from experimental result. Therefore, these simulations prove that high NI currents can be extracted with e/NI current ratio less than 1 if and only if a significant fraction of the surface produced NI is direct extracted.

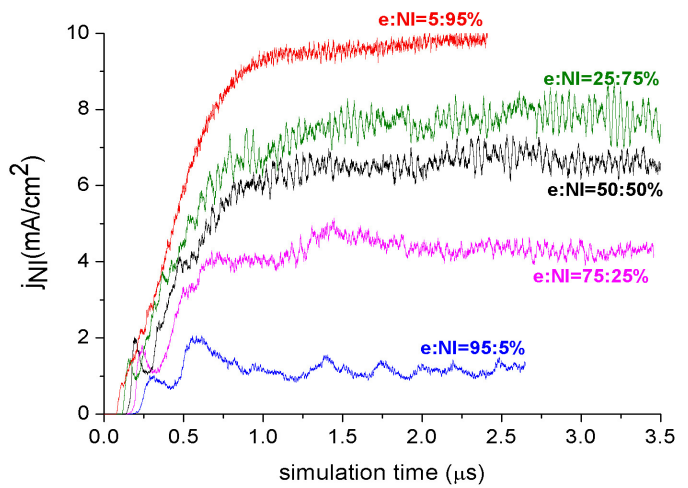

FIG. 5 NI volume extracted current density from parametric study of different initial bulk plasma.

\section{CONCLUSIONS}

The ONIX code is applied at IPP Garching in order to perform realistic simulation and direct cross-checked analysis with experimental results. The code has been validated and benchmarked with other 3D numerical model KOBRA3D for PI extraction with very good agreement in terms of the "meniscus" shape. The parametric study of e/NI density ratio in the bulk plasma shows the high relevance of the direct NI extraction from PG surface.

${ }^{1}$ R. Hemsworth, H. Decamps, J. Graceffa, B. Schunke, M. Tanaka, M. Dremel, A. Tanga, H.P.L. De Esch, F. Geli, J. Milnes, T. Inoue, D. Marcuzzi, P. Sonato and P. Zaccaria, Nucl. Fusion, 49, 045006, 2009. ${ }^{2}$ L. Schiesko, P. Franzen and U. Fantz, Plasma Sources Sci. Technol., 21, 065007, 2012.

${ }^{3}$ Y. Belchenko, Rev. Sci. Instrum., 64, 1385, 1993.

${ }^{4}$ E. Speth H.D. Falter, P. Franzen, U. Fantz, M. Bandyopadhyay, S. Christ, A. Encheva, M. Fröschle, D. Holtum, B. Heinemann, W. Kraus, A. Lorenz, Ch. Martens, P. McNeely, S. Obermayer, R. Riedl, R. Süss, A. Tanga, R. Wilhelm and D. Wünderlich, Nucl. Fusion , 46, S220, 2006.

${ }^{5}$ B. Heinemann, H. Falter, U. Fantz, P. Franzen, and M. Fröschle, Fusion Eng. Des., 84, 915, 2009.

${ }^{6}$ S. Mochalskyy, A.F. Lifschitz and T. Minea., Nucl. Fusion, 50, 105011, 2010 .

${ }^{7}$ S. Mochalskyy, A.F. Lifschitz and T. Minea., J. Appl. Phys., 111, $113303,2012$.

${ }^{8}$ C.K. Birdsall and A.B. Langdon, "Plasma Physics via Computer Simulation" (1985).

${ }^{9}$ D. Wünderlich et al., Plasma Sources Sci. Technol., 18, 045005, 2009

${ }^{10}$ P. Spädke and S. Wipf, Technical report GSI-89-09, 1989.

${ }^{11}$ M. Berger et U. Fantz, S. Christ-Koch and NNBI Team, Plasma Sources Sci. Technol., 18, 025004, 2009. 